University of Wollongong

Research Online

Australian Institute for Innovative Materials -

Papers

Australian Institute for Innovative Materials

$1-1-2018$

Surface modification of polyaniline nanorods with thiol-terminated poly(ethylene oxide)

Brandon DiTullio

Furman University

Cassandra J. Wright

University of Washington

Patricia Y. Hayes

University of Wollongong, phayes@uow.edu.au

Paul J. Molino

University of Wollongong, pmolino@uow.edu.au

Timothy W. Hanks

Furman University, tim.hanks@furman.edu

Follow this and additional works at: https://ro.uow.edu.au/aiimpapers

Part of the Engineering Commons, and the Physical Sciences and Mathematics Commons

Research Online is the open access institutional repository for the University of Wollongong. For further information contact the UOW Library: research-pubs@uow.edu.au 


\title{
Surface modification of polyaniline nanorods with thiol-terminated poly(ethylene oxide)
}

\begin{abstract}
Electrochemically grown polyaniline (PAni) thin films have been shown to react efficiently with thiols, which can dramatically change the surface properties of the material without significantly impacting bulk conductivity. Such films, however, are difficult to process and are unsuitable for many applications. Here, we demonstrate the grafting of thiol-terminated poly(ethylene oxide) (PEG-SH) of various molecular weights onto PAni nanorods. The resulting materials are characterized by spectroscopic, microscopic, and thermal analytical methods to demonstrate the covalent attachment of the PEG polymers to the nanorods. The derivatized nanorods are water dispersible and maintain their original morphology and electroactivity. The number of thiols bound to the nanoparticles under a given set of conditions decreases as the size increases, but the total mass of PEG increases with increasing size. The reaction proceeds at room temperature, but is much faster at higher temperature and greater PEG density is observed.

\section{Disciplines}

Engineering | Physical Sciences and Mathematics

\section{Publication Details}

DiTullio, B., Wright, C. J., Hayes, P., Molino, P. J. \& Hanks, T. W. (2018). Surface modification of polyaniline nanorods with thiol-terminated poly(ethylene oxide). Colloid and Polymer Science: Kolloid-Zeitschrift und Zeitschrift fuer Polymere, 296 (4), 637-645.
\end{abstract}




\title{
Surface modification of polyaniline nanorods with thiol-terminated poly(ethylene oxide)
}

\author{
Brandon T. DiTullio ${ }^{1}$ - Cassandra J. Wright ${ }^{2} \cdot$ Patricia Hayes $^{3} \cdot$ Paul J. Molino $^{3,4} \cdot$ Timothy W. Hanks $^{1}$
}

Received: 28 November 2017 / Revised: 14 January 2018 / Accepted: 24 January 2018 / Published online: 15 February 2018

(C) Springer-Verlag GmbH Germany, part of Springer Nature 2018

\begin{abstract}
Electrochemically grown polyaniline (PAni) thin films have been shown to react efficiently with thiols, which can dramatically change the surface properties of the material without significantly impacting bulk conductivity. Such films, however, are difficult to process and are unsuitable for many applications. Here, we demonstrate the grafting of thiol-terminated poly(ethylene oxide) (PEG$\mathrm{SH}$ ) of various molecular weights onto PAni nanorods. The resulting materials are characterized by spectroscopic, microscopic, and thermal analytical methods to demonstrate the covalent attachment of the PEG polymers to the nanorods. The derivatized nanorods are water dispersible and maintain their original morphology and electroactivity. The number of thiols bound to the nanoparticles under a given set of conditions decreases as the size increases, but the total mass of PEG increases with increasing size. The reaction proceeds at room temperature, but is much faster at higher temperature and greater PEG density is observed.
\end{abstract}

Keywords Polyaniline $\cdot$ Poly (ethylene oxide $) \cdot$ Thiol $\cdot$ Nanoparticle $\cdot$ Surface modification

\section{Introduction}

Nanostructured, intrinsically conducting polymers (ICPs) are of considerable current interest due to their role in a host of composite materials $[1,2]$. A valuable feature of ICPs in this regard is that the physical, chemical, electrical, and mechanical properties of ICPs can be modified by several methods [3, 4]. The most obvious of these are to chemically derivatize the monomers or to change nature of the counterion (commonly called the "dopant") used to balance the polyionic backbone. Both of these methods will profoundly affect the electronic

Timothy W. Hanks tim.hanks@furman.edu

1 Department of Chemistry, Furman University, 3300 Poinsett Hwy, Greenville, SC 29613, USA

2 Division of Engineering and Mathematics, University of Washington Bothell, 18115 Campus Way NE, Bothell, WA 98011-8246, USA

3 ARC Centre of Excellence for Electromaterials Science, Intelligent Polymer Research Institute/AIIM Faculty, University of Wollongong, Innovation Campus, Squires Way, Wollongong, NSW 2522, Australia

4 ARC Research Hub for Australian Steel Manufacturing, Intelligent Polymer Research Institute/AIIM Faculty, University of Wollongong, Innovation Campus, Squires Way, Wollongong, NSW 2522, Australia and physical properties of the material. A less common method for ICP modification is to alter only the surface of a polymeric object, leaving the bulk properties unaffected.

Of the ICPs, polyaniline (PAni) is of particular interest in applications such as biosensing [5], bioelectronics [6], tissue engineering [7, 8], corrosion protection [9], anti-static packaging [10], organic light emitting diodes (OLEDs) [11], and other electronic or optoelectronic devices [12]. PAni not only has useful electronic properties, but it is easily prepared from inexpensive reagents, making large scale production feasible [13]. Additionally, PAni has excellent electrochemical and environmental stability [14]. In some cases, ICPs have been reported to suffer from poor thermal stability in air and they are often reduced to a non-conductive state in aqueous solutions, limiting long-term usage in physiological environments [15]. In contrast, PAni maintains conductivity and structural properties in the presence of both oxygen and water vapor rich air. The judicious choice of dopant and additives can further improve upon this stability [16].

There are two general strategies for PAni polymerization: chemical and electrochemical. Electrochemical polymerizations are preferred if the polymer is intended for use in polymer electrodes, thin-layer sensors, in many electronic applications, and so on. This is because control of oxidizing potential during polymerization allows for the production of highly organized architectures and growth of the film to controlled thicknesses. 
Electrochemically grown PAni is necessarily limited to small quantities of material deposited on an electrode surface. The films are insoluble and generally not suitable for further processing.

The chemical polymerization of aniline is a simple process that can readily produce large quantities of product. Traditionally, this material is formed as an insoluble powder with limited processability. However, more recent work has allowed for the formation of other morphologies, including electrospun fibers, hydrogels, and nanoparticles. Recent syntheses to produce nanorod forms of PAni have been shown to be a material with superior tunability and improved processability due to its increased surface area, while maintaining its intrinsic conductivity [17]. PAni nanorods can be processed as self-stabilized colloids, which can then be chemically or electrochemically engineered, allowing the tuning of its physicochemical properties. Furthermore, the nanofibrous form has been shown to increase a material's surface roughness, and used to enhance the wettability of surfaces [18].

Here, we explore the surface functionalization of PAni nanorods as a strategy for fine-tuning their physical and chemical properties and improving their usefulness in both physiological and biological environments [19]. In a previous study, we demonstrated that the surfaces of electrochemically grown PAni thin films were readily modified by exposure to thiolterminated poly(ethylene oxide) (PEG-SH [20]). PEGs have a low interfacial free energy in water and extended hydrophilic PEG chains have been shown to provide steric stabilization of nanoparticles [21]. The neutral polyether is also notable for its facility in repelling other polymers (such as proteins, lipids, and nucleic acids) while in an aqueous environment. Appropriately end-functionalized PEG chains have grafted or attached to various surfaces to impart this fouling resistance. Additionally, it has been reported that the chemical modification of nanoparticles with PEG results in more controlled solubility [22].

Here, we demonstrate the functionalization of the surface of PAni nanorods while maintaining the intrinsic characteristics of the original aniline polymer using a solution-based chemical modification approach. The resulting PAni nanorods retain their $\mathrm{pH}$ sensitivity, electrical conductivity and redox properties as well as their nanomorphology and good thermal stability. The method presented herein describes a convenient, inexpensive, and scalable route to PAni-PEG composite nanomaterials.

\section{Materials and methods}

\section{Materials}

Aniline was purchased from Sigma-Aldrich, purified by distillation, and stored at $-2{ }^{\circ} \mathrm{C}$ until used. Varying molecular weights (1k, 5k, 20k, 40k) of poly(ethylene) glycol methyl ether thiol (PEG-SH) were purchased from JenKem Technology and used as received. All other reagents were purchased from commercial houses and used as received. Deionized water was purified using a Millipore Ultrapure water purification system.

\section{Nanorod preparation}

The nanorods were prepared by a modification of a route reported by Huang and Kaner [23]. Ammonium persulfate $(2.90 \mathrm{~g}, 12.7 \mathrm{mmol})$ was dissolved in $500 \mathrm{~mL}$ of $1 \mathrm{M} \mathrm{HCl}$ with the aid of mechanical stirring. Aniline $(4.9 \mathrm{~g}, 52.6 \mathrm{mmol})$ was added to $500 \mathrm{~mL}$ of toluene and the two solutions were then carefully combined at ambient temperature without agitation. After $24 \mathrm{~h}$ at room temperature, nanorods were isolated using centrifugation. The nanorods were re-dispersed in DI water with the aid of sonication and isolated by centrifugation three times. They were likewise re-suspended and collected in acetone. Finally, they were dried for $12 \mathrm{~h}$ at $40{ }^{\circ} \mathrm{C}$ in vacuo. The PAni nanorods were stored under nitrogen until used.

\section{Nanorod film preparation}

The dispersant used for PAni processing in this study consisted of a 50:50 water/ethanol solution. Homogenous, thin PAni films were produced by spin coating low concentrated solutions $(<0.1$ PAni wt $\%)$ onto the desired substrate. Thicker and rougher films were made by drop casting higher concentrations ( $>0.5 \%$ PAni wt $\%$ ) onto the surfaces.

\section{Functionalization of nanorods}

PAni nanoparticles $(20 \mathrm{mg})$ were added to $15 \mathrm{~mL}$ of DI water (pH 5.5). The PAni mixture was sonicated using a Branson Digital Sonifier and a model 102-C Converter. Mixtures were sonicated for approximately $10 \mathrm{~min}$ to help disperse the nanorods and to increase the surface area available for the PEGthiol reaction. Varying quantities and sizes (depending on the specific study) of PEG-thiol were then dissolved in $15 \mathrm{~mL}$ of DI water in a separate vial. The PAni and PEG solutions were then combined and allowed to react at either room temperature or at reflux. The resulting product was collected by centrifugation at 10,000 rpm for $30 \mathrm{~min}$ and washed/isolated three times with DI water. The supernatant from the washes and the final products were analyzed by ${ }^{1} \mathrm{H}-\mathrm{NMR}$ to ensure the purification methods removed unreacted PEG-thiol and PEGdisulfide (an impurity always present in the reagent). A freeze dryer was used to remove the remaining water from the modified nanorods. The final product proved to be very hygroscopic and was therefore stored in a desiccator at room temperature in vacuo. 


\section{Nanorod characterization}

The derivatized nanorods were characterized by various spectroscopic, microscopic, and thermal methods. ${ }^{1} \mathrm{H}-\mathrm{NMR}$ data was collected at $30{ }^{\circ} \mathrm{C}$ on a $400 \mathrm{MHz}$ Bruker Advance III spectrometer in $\mathrm{d}^{6}$-DMSO. The sample was passed through a $0.2-\mu \mathrm{m}$ filter to remove aggregates. The spectra were calibrated to the residual solvent peak $(\delta=2.50 \mathrm{ppm})$. Details of the degree of functionalization were obtained with a TA Instruments Q500 thermogravimetric analyzer. The ramp and final temperature in all TGA measurements were set to $10{ }^{\circ} \mathrm{C} / \mathrm{min}$ and $1000{ }^{\circ} \mathrm{C}$, respectively. TGA spectra are representative of the raw data for any reaction conditions tested. Any averages provided are based on triplicate measurements and mean variance was not determined to be significant for any of the data populations. UV-Vis spectroscopy was carried out using a Varian Cary 50 probe UV-Visible spectrometer. The electrochemical properties were studied using an Epsilon Electrochemical Analyzer power source and software with a BASi C3 cell. Cyclic voltammetry was conducted with a three-electrode arrangement using a $0.1-\mathrm{M} \mathrm{KCl}$ electrolyte at $\mathrm{pH} 1$ with a scan rate of $10 \mathrm{mV} / \mathrm{s}$. All CV curves were obtained by scanning between -0.2 and $0.9 \mathrm{~V}$ (vs SCE). A gold disk working electrode, a Pt wire counter-electrode, and a $\mathrm{Ag} / \mathrm{AgCl}$ reference electrode were used. Samples were prepared by drop casting dispersed nanorods onto a polished gold working electrode, which were left in a vacuum oven at $70^{\circ} \mathrm{C}$ for $10 \mathrm{~min}$ to dry. Morphological data were obtained using a JSM-7100F field emission scanning electron microscope at an accelerating voltage of $15 \mathrm{kV}$. Sample preparation consisted of casting control and derivatized PAni nanorods onto high-quality magnetic stainless steel (alloy 430) discs.

\section{Results and discussion}

\section{General}

PAni nanorods were prepared by slow growth at a $\mathrm{HCl} /$ toluene interface as previously described [23]. Modified PAni nanorods used for spectroscopic characterization were prepared by treating $20 \mathrm{mg}$ of PAni nanorods with an aqueous solution of $50 \mathrm{mg}$ of a $1000 \mathrm{MW}$ PEG-SH by either stirring for $24 \mathrm{~h}$ at room temperature or heating at reflux for $2 \mathrm{~h}$. The nanorods were purified by twice re-suspending in DI water and isolating by centrifugation.

The nanorods could be drop cast, spin-coated, or spraycoated depending on the type of film topography desired. This was useful in performing cyclic voltammetry and scanning electron microscopy analysis on the PAni nanorods and its derivatives. The concentration of the polymer in the dispersant played a significant role in the homogeneity and thickness of the films achieved after deposition. Casting lower concentrated solutions ( $<0.1$ PAni wt $\%$ ) yielded surfaces with much more homogeneous, thin films. Coatings made using higher concentrations $(>0.5 \%$ PAni wt $\%$ ) produced much thicker and rougher films.

Previous reports have indicated that $\pi$-conjugated molecules form regular nanostructured morphologies and aggregate structures through hydrogen bonding and $\pi$-stacking interactions [24]. The aggregations that these non-covalent atomic and molecular attractions formed in solution can be controlled by the dopant and the solvent system used to disperse them, as the degree of aggregation of the aniline polymer varies dramatically. Tuning the solvent/solvent ratio further allows the extent of aggregation to be controlled [25]. Solvent systems with a higher polar component enhance the $\pi$-stacking interactions, which increases the amount of aggregation. This was considered when choosing an ideal solvent system to work with in polyaniline thin film preparation.

\section{Effect of reaction temperature}

The nanorod derivatization reaction was conducted at room temperature and at reflux in order to investigate the reaction efficiency as a function of temperature. Thermogravimetric analysis offered a reliable method of quantifying the amount of PEG-SH addition to polyaniline (Fig. 1). The PEG-SH decomposes with an onset temperature of $351^{\circ} \mathrm{C}$, while the PANI nanorods, after an initial loss of adsorbed water, showed a decomposition onset of $501^{\circ} \mathrm{C}$. The room temperature reaction proved to be slow and after 24 the product contained $15 \%$ of PEG-SH $(\mathrm{MW}=1000)$ by mass. When the reaction was run under reflux for $2 \mathrm{~h}$, the PEG-SH content increased to $27 \%$ by mass.

In a related experiment, PANI was reacted with PEG-SH $(\mathrm{MW}=5000)$ at elevated temperature and monitored with time (Fig. 2). Again, elevated temperatures increased the extent of reaction over a room temperature control. The amount of thiol added to the nanoparticles increased with time, but showed only a modest increase in between 2 and $4 \mathrm{~h}$. As the PEG content of the nanorods increases, the onset of decomposition of the PEG components increased from 291 to $321{ }^{\circ} \mathrm{C}$. These results are consistent with previous work on inorganic surfaces where PEG grafting density has been shown to be sensitive to reaction temperature [26]. Higher temperatures result in increased PEG solubility and chain extension into solution, providing increased access to the PANI surface.

\section{Electronic properties}

The electronic properties of the nanorods depend on the degree of thiol derivatization. As with other nucleophiles, the reaction between PEG-SH and PAni results in the partial reduction of the aniline rings, probably through the mechanism 
Fig. 1 Thermal gravimetric analysis of PEG-SH (MW = 1000) control, PAni nanorods derivatized with PEG-SH at room and elevated temperatures and unmodified PAni nanorods

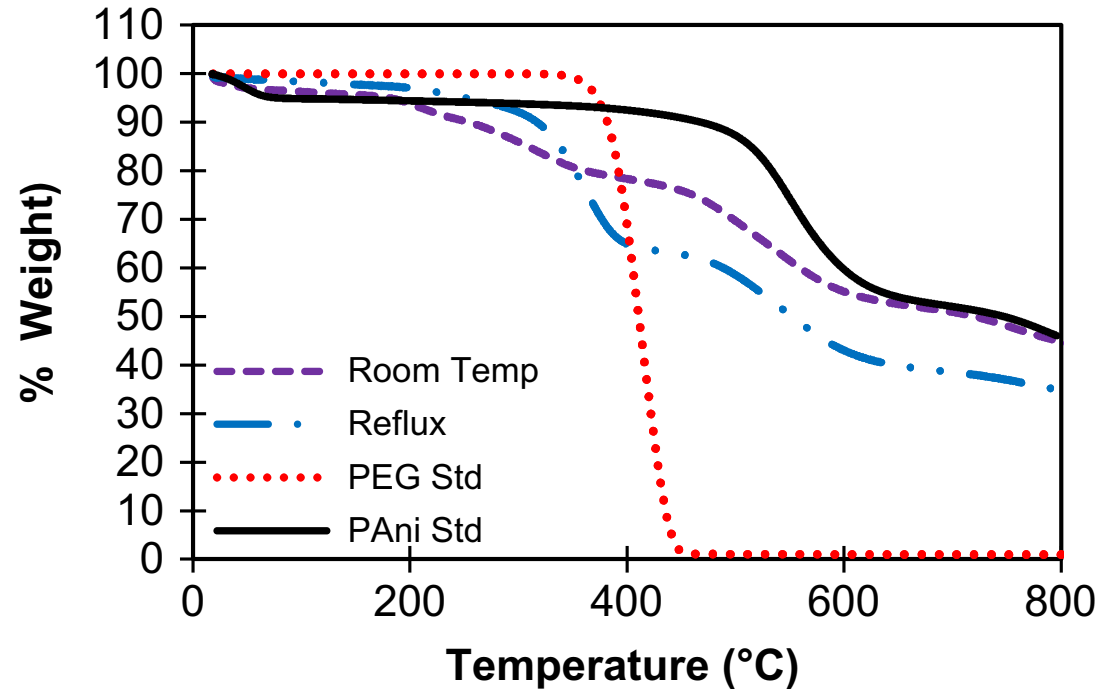

illustrated in Scheme 1 [27]. Not all of the PAni diiminoquinoid rings are reduced, probably because the interior of the thiol is unable to penetrate deeply into the nanorods.

UV-visible spectroscopy was used to examine effect of derivatization on the nanofiber optical properties, as influenced by the aniline ring conjugation and conformation (Fig. 3). The PAni-emeraldine base (EB) absorbances at 430 and $800 \mathrm{~nm}$ correspond to the $\pi-\pi^{*}$ transition of the benzenoid structures and the absorption of the excitons (polaron transitions) in the quinoid rings, respectively [28]. A bathochromic shift for these bands is observed in the more highly derivatized product from the elevated temperature reaction, which is due to the increased electron density from the sulfur substituents. The small absorbance at $340 \mathrm{~nm}$, which is present in the PEG-PAni but absent in the unmodified PAni, is caused by a $\pi-\pi^{*}$ transition induced by quinoid ring substitution. This absorbance intensifies with increasing degree of

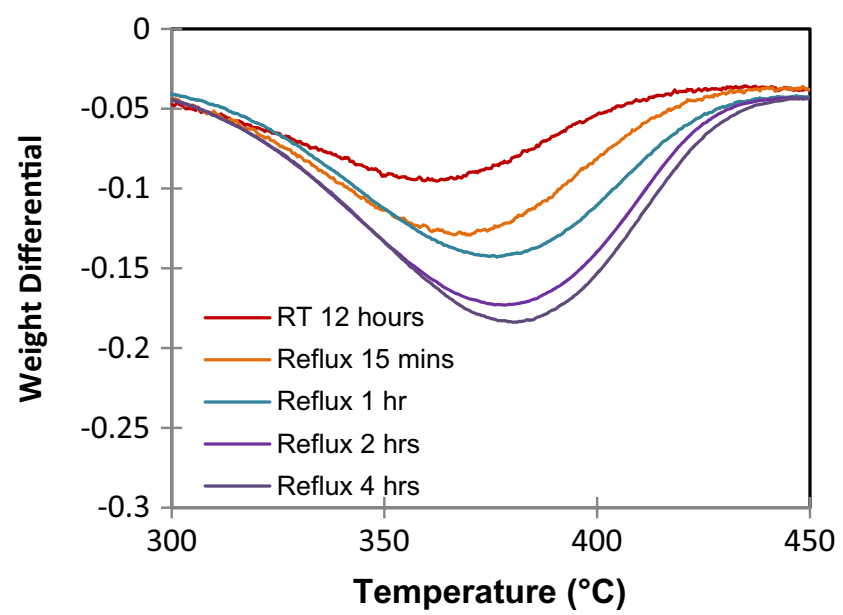

Fig. 2 Derivative TGA plot shows the extent of PEG loss from decomposition from the PAni-PEG nanorods as the thiol derivatization reaction conditions were changed modification as the reduction breaks up the extended conjugation.

As with PANI, the derivatized nanorods can be reversibly protonated with acid to transform them into an emeraldine/ leucoemeraldine salt state, which is indicated by an increase in the polaron band intensity at the expense of the $\pi-\pi^{*}$ transition. As shown in Fig. 4, the resulting green product can be deprotonated with a base to give a blue color.

While bipolaron states are found in PAni, the primary charge carriers in the emeraldine salt form are polarons. This is due to the presence of coulombic interactions, local disorder in PAni structural lattice, and dielectric screening, which all act to stabilize the energetically unfavorable, delocalized polaron state [29]. These polaron transitions are strongly related to the conductivity of the bulk material. This suggests that while the modified material does maintain electron transport properties of the parent PAni, there is a decrease as the degree of functionalization increases. This is expected due to the loss in long chain $\pi$-conjugation from the increased ring substitution. Reoxidation, however, largely restores the conductivity.

\section{Electron microscopy}

Modified nanorods were prepared by treating them with a 2.5fold excess (by weight) of 1000K MW PEG-SH in water and heating at reflux for $2 \mathrm{~h}$ and then cast as thin films from colloidal dispersions. Figure 5 shows the morphologies of modified and unmodified nanorods. The nanorods were 50$100 \mathrm{~nm}$ in diameter and several hundred nanometers in length as measured by SEM (Fig. 5). The SEM images show that the reaction conditions did not damage the nanorods, with the major difference in the images being the extent of charging. This is consistent with the thiol reaction causing a reduction in the ability of the nanorods to dissipate charge build-up from the electron beam. Conversely, Lahiff found that reaction with small molecular thiols resulted in an increase in surface 
Scheme 1 Proposed mechanism of the reaction of thiols with PAni

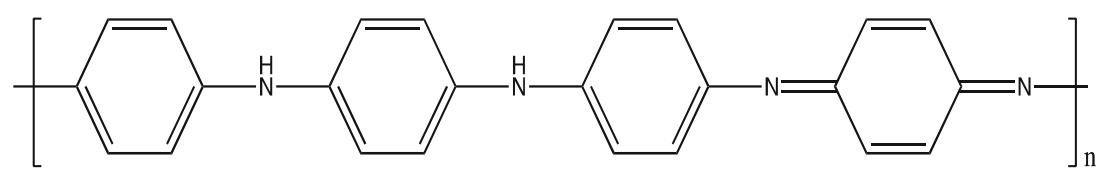

Emeraldine Base

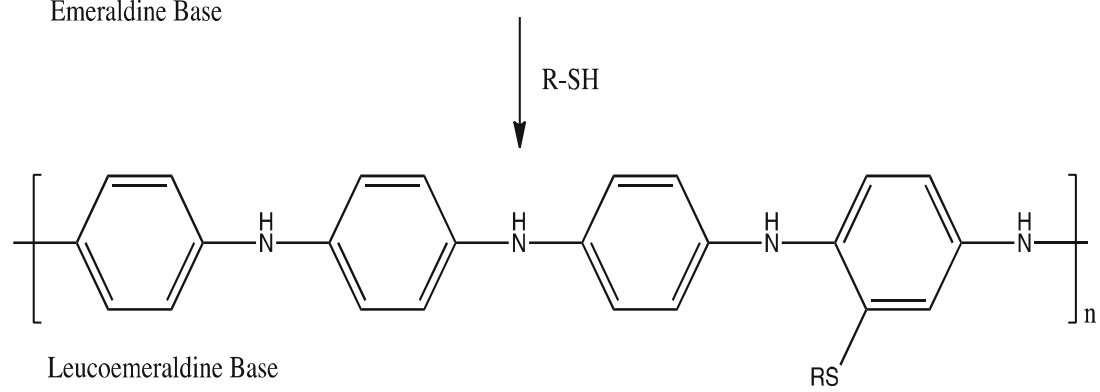

roughness of similar PAni nanorods with increasing degree of derivatization [30].

\section{${ }^{1}$ H-NMR}

Key evidence of nucleophilic substitution on some of the quinoid rings of the PAni backbone was obtained from ${ }^{1} \mathrm{H}$ NMR data of the product suspension in $\mathrm{d}^{6}$-DMSO. The ${ }^{1} \mathrm{H}-$ NMR spectra of PEG-SH and PAni-PEG are shown in Fig. 6. Not shown in this expansion are the aromatic protons around $7 \mathrm{ppm}$ in the PAni-PEG spectrum. The weak triplet peak observed at $\delta 2.90 \mathrm{ppm}$ in the PEG-SH standard spectrum was determined to be the disulfide formed by oxidation of the PEG-SH in the starting material. Integration of the signal shows that there is approximately $6.5 \%$ of an impurity identified as the symmetrical disulfide of PEG-SH. This is consistent with the certificate of analysis for PEG-SH (SigmaAldrich, 729108, Lot number: MKBD6808V) indicating the presence of $7.3 \%$ of the disulfide (determined by GPC).

In the modified nanorods, the chemical shift observed for the signal corresponding to the methylene group adjacent to

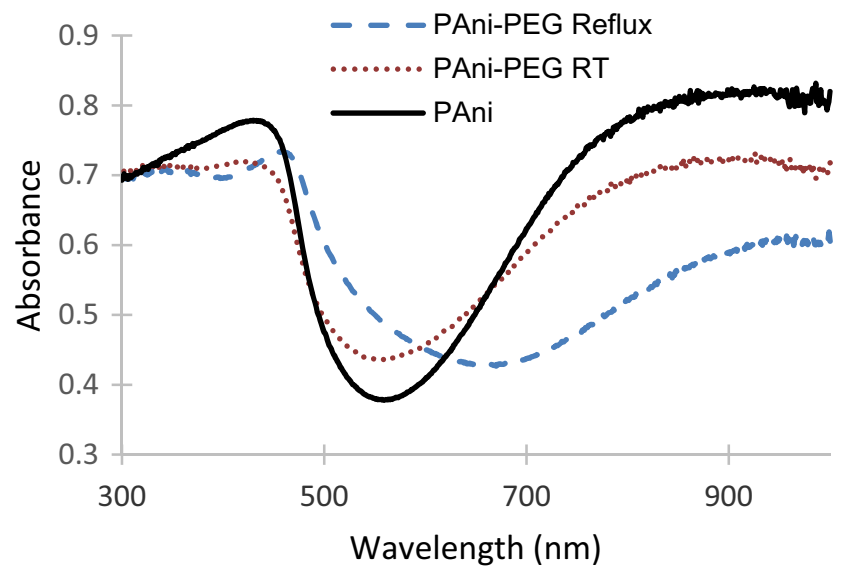

Fig. 3 UV-vis spectra of PANI nanorods. a Unmodified. b Modified at room temperature. $\mathbf{c}$ Modified at reflux the sulfur bond $\left(\mathrm{CH}_{2}-\mathrm{S}\right)$ shifts from $\delta 2.62(\mathrm{dt}, J 6.8,8.0 \mathrm{~Hz})$ in PEG-SH to $\delta 2.90(t, J 6.4 \mathrm{~Hz})$, consistent with the contention covalent attachment had occurred. These thioether peaks are similar to those of previously reported thiols covalently bound to polyesters [31] and small molecular weight thiols bound to PAni [30, 32]. Additional evidence for covalent attachment is provided by the loss of the coupling $(J 8.0 \mathrm{~Hz})$ to the thiol proton in PAni-PEG (triplet instead of doublet of triplet) and by the disappearance of the thiol proton signal at $\delta 2.25(t, J$ 8.0 Hz, SH in PEG-SH) in the proton spectrum of PAni-PEG nanorods. No evidence of unreacted thiol could be observed.

The purification process, consisting of repeated suspension of the derivatized nanorods followed by centrifugation, leaving a supernatant that contains only the disulfide PEG-S-SPEG and a trace of PEG-SH. While disulfide production may be the partially due to oxidation from a small amount that oxygen entering the reaction vessel, the principle route is a redox process with the PAni that competes with the thiol addition. We have previously observed this reaction during the addition of simple thiols to polythiophene, where disulfide

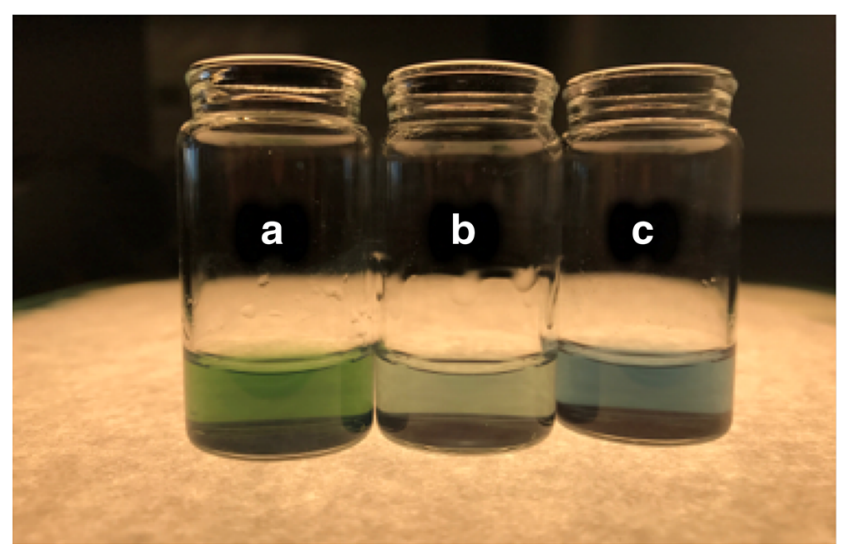

Fig. 4 Equal amounts of modified PAni nanorods dispersed in a $1 \mathrm{M}$ $\mathrm{HCl}$, b DI water, and $\mathbf{c} 1 \mathrm{M} \mathrm{NH}_{3}$ 
Fig. 5 FESEM images of PAni nanorods unmodified (left) and modified (right) at a loading of $25 \%$ PEG-SH $w / w$
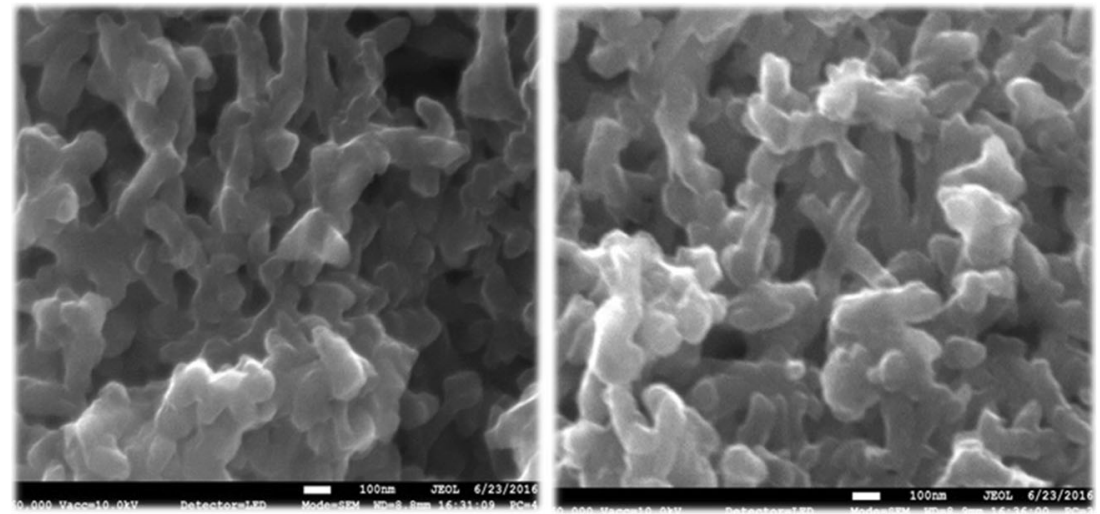

production is the dominant pathway and to a lesser extent with poly(3,4-ethylenedioxythiophene) and polypyrrole [33].

\section{Cyclic voltammetry}

The electrochemical behavior of the modified PAni nanorods was studied using cyclic voltammetry $(\mathrm{CV})$. It is well known that electrochemically active PAni has three distinct oxidation states with varying physicochemical properties (pernigraniline, emeraldine, and leucoemeraldine) [34, 35]. Figure 7 shows the voltammograms of PAni treated with PEG-SH $(\mathrm{MW}=1000)$ at high and low temperatures vs a PAni control. Under acidic conditions, the PAni nanorods show a broad redox couple at 0.23 and $0.10 \mathrm{mV}$ and a second, sharper couple at 0.82 and $0.60 \mathrm{mV}$. The more highly substituted materials from the high temperature reaction showed dramatic changes, including a shift of the first redox couple to more positive potential and the second to lower potential. In addition, a third redox couple appears at intermediate potentials and the second and third events become less well defined.

The room temperature reaction, with a relatively low level of functionalization is very similar to the PAni nanorod control, with a small shift of the redox events due to the introduction of the thiols on a portion of the PAni rings. The reason(s) for the electrochemical changes causing these shifts are not fully understood, but are similar to those that have been observed previously in related systems [36, 37]. The partial derivatization of the thiols results in individual PAni chains with different degrees of substitution with an electron-donating group, leading to a less homogeneous material. The introduction of the nonconducting PEG moiety will also affect the ease of counterion transport into and out of the films.
Fig. $6{ }^{1} \mathrm{H}-\mathrm{NMR}\left(400 \mathrm{Mhz}, \mathrm{d}_{6}\right.$ DMSO) of PAni-PEG final product (top, $\mathrm{MW}=1000$ ) and PEG-SH standard, bottom. Rectangles highlight the resonances of the methylene protons immediately adjacent to the sulfur. The circle shows to the thiol proton, which is not seen in the PAni-PEG

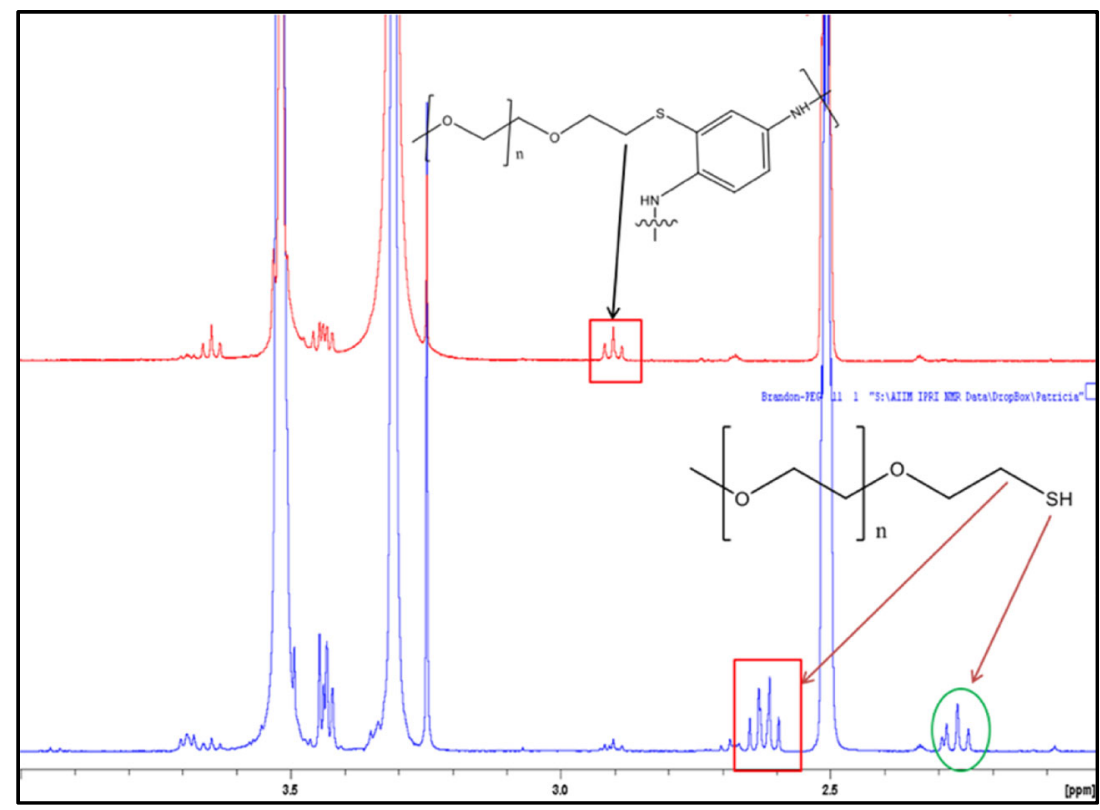




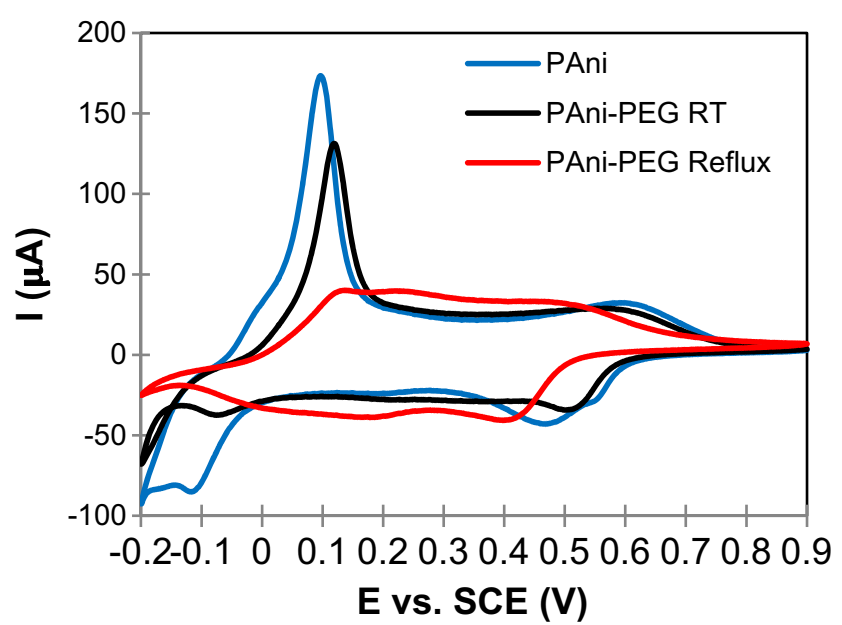

Fig. 7 CV curves for PAni nanorods drop cast onto a gold disk electrode. Shown are pristine nanorods and rods treated with PEG-SH $(M W=1000)$ at room and reflux temperatures

\section{Effect of thiol concentration}

Thiol/PAni ratios are an important parameter to control when considering this chemical functionalization method. Lahiff et al. reported complete deterioration of PAni nanostructures when exposed to large concentrations of mercaptoundecanoic acid (MA-SH), particularly at MA-SH/PAni ratios of greater than $5: 1$ by mass [30]. This deterioration was ascribed to either the high concentration of acidic carboxylic acid functional groups interfering with the H-bonding between PAni backbones, or the over-extension of the PAni backbones as a result of steric repulsion.

When comparing the PEG-SH with the MA-SH used in the aforementioned study, a few differences are notable in regard to the potential effect on PAni nanostructures. First, the molecular weights of the PEG-SH $(1 \mathrm{~K}, 5 \mathrm{~K}, 20 \mathrm{~K}$, and $40 \mathrm{~K})$ used in this investigation are much larger than the MA-SH used in the Lahiff study, which had a molecular weight of about 220. Thus, the steric consequences for the addition of each molecule of thiol are much more significant. Conversely, it means that there are fewer C-S bonds formed for an equivalent amount of mass added to the PAni. We would also expect that these larger thiols will also have more difficulty penetrating into the nanorods, because their lower intrinsic diffusivity and steric bulk limits penetration into nanorod pores and because the initial thiols deposited physically block access to later reactants.

It has been shown that the degree of covalent sidechain attachment to PAni increases as a function of the thiol concentration and this was found to be the case with this system as well $[30,32]$. Equal quantities of PAni nanorods $(20 \mathrm{mg})$ were surface derivatized with increasing amounts of $5,10,20,50$, and $200 \mathrm{mg}$ of $5000 \mathrm{mo}-$ lecular weight PEG-SH at reflux, as shown in Table 1.
Table 1 The effect of PEG-SH concentration on incorporation in to PAni-PEG composites. PEG-SH $(\mathrm{MW}=5000)$ and PAni $(20 \mathrm{mg})$ dispersed in water and heated to reflux for $4 \mathrm{~h}$

\begin{tabular}{cl}
\hline PEG-SH added (mg) & PEG-SH incorporation (\%) \\
\hline 5 & 12.3 \\
10 & 15.3 \\
20 & 19.4 \\
50 & 21.3 \\
200 & 30.2 \\
\hline
\end{tabular}

TGA showed that the amount of thiol incorporated into the final product after thoroughly washing steadily increased. As in Fig. 2, the temperature of maximum decomposition increased with increasing PEG incorporation.

This is most likely due to the PEG resembling the thermal behavior of the free 5k MW PEG-SH, which has a thermal decomposition onset temperature of c.a. $400{ }^{\circ} \mathrm{C}$. Additionally, it may be due to a higher degree of heat transfer associated with the PAni relative to the PEG. With the lower derivatized products, the lower IDTs are observed, which provides evidence that the clean PEG polymer has a lower heat transfer than that of the PAni. This study provides strong evidence that the ratio of PEG-SH to PAni is an important factor to consider when attempting to control the degree of functionalized PAni nanorods.

\section{Effect of thiol molecular weight}

The PAni nanorods were functionalized with various molecular weight PEG-thiols to determine if there is a difference in the nucleophilic loading or binding density as a function of the thiol polymer chain length. The molar amounts of PEG-thiol molecules were kept constant between all four molecular weights tested $(1 \mathrm{~K}, 5 \mathrm{~K}, 20 \mathrm{~K}, 40 \mathrm{~K})$ to

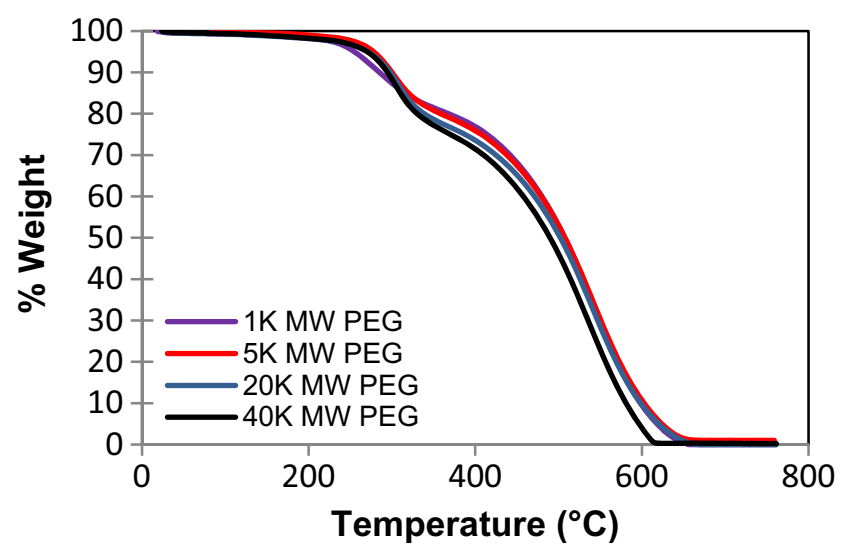

Fig. 8 TGA data showing the weight percent of PAni and PEG components made from varying PEG-SH molecular weights 
prevent the number of nucleophilic sulfur groups from being a variable. Duplicate experiments with 10, 50, 200 , and $400 \mathrm{mg}$ of $1 \mathrm{~K}, 5 \mathrm{~K}, 20 \mathrm{~K}$, and $40 \mathrm{~K} \mathrm{MW}$ PEG-SH $(10 \mu \mathrm{mols})$ were used, respectively. These were reacted with $20 \mathrm{mg}$ of PAni in each case.

We have shown previously with PAni thin films that as the size of the PEG-SH increases, the total mass of PEG covalently bound also increases [38]. Not surprisingly, similar results were found in this study, as shown in Fig. 8.

The onset temperatures of thermal decomposition for PEG-SH and PAni were determined to be approximately 300 and $420{ }^{\circ} \mathrm{C}$, respectively. However, as shown in Fig. 8, the initial degradation temperature of PEG increases as a function of molecular weight. Again, the most probable justifications for this trend are a better heat transfer of the PANI component PAni compared to PEG, resulting in materials with a higher PEG weight fraction PAni displaying thermal behavior closer to that of the original PEG standard material.

By integrating the TGA decomposition peaks in Fig. 8, we were able to determine the fractional weight percent of adsorption for each molecular weight PEG reacted with the PAni nanorods: $1 \mathrm{~K}(10.3 \%), 5 \mathrm{~K}(13.9 \%), 20 \mathrm{~K}(14.8 \%)$, and $40 \mathrm{~K}$ $(19.8 \%)$. The resulting PEG/PAni molecular ratios were $1 / 109,1 / 388,1 / 1447$, and $1 / 2171$ for the $1 \mathrm{~K}, 5 \mathrm{~K}, 20 \mathrm{~K}$, and 40K molecular weight PEGs, respectively. These numbers indicate that the bonding efficacy of covalent attachment onto PAni nanorods significantly decreases with increasing molecular weight. However, as shown by the percent weight composition values above, there is an overall increase in PEG weight deposition as a function of the molecular weight PEG-SH used.

\section{Conclusions}

PEG-derivatized PAni nanorods were synthesized by a facile solution surface modification process. Bulk quantities of PAni nanorods were refluxed to form functionalized material in high yields, which indicates this technique is easily scalable. The degree of PAni derivatization can be easily controlled by controlling the reaction temperature conditions and the ratio of PEG-SH to PAni. It has been shown that PAni-PEG maintain the $\mathrm{pH}$ sensitivity, spectroscopic, conductivity, and electroactive properties of the PAni nanorods.

Acknowledgements Acknowledgment is made to the Donors of the American Chemical Society Petroleum Research Fund for support of this research (Grant \# 54873-UR7). BD thanks the Furman Advantage program for a fellowship. CW was supported by SC-INBRE PACD fellowship; grant \# P20GM103499 from the National Institute of General Medical Sciences. Analytical support from the Australian National Nanofabrication Facility and the UOW Electron Microscopy Centre is acknowledged.
Funding This study was funded by the American Chemical Society Petroleum Research Fund (Grant \# 54873-UR7). Author DiTullio was supported by a summer undergraduate fellowship from Furman University. Author Wright was supported through a SC-INBRE PACD fellowship as part of grant \# P20GM103499 from the National Institute of General Medical Sciences.

\section{Compliance with ethical standards}

Conflict of interest The authors declare that they have no conflict of interest.

\section{References}

1. Ćirić-Marjanović G (2010) In: Eftekhari A (ed) Nanostructured conductive polymers. Wiley, Chichester

2. Nguyen DN, Yoon H (2016) Recent advances in nanostructured conducting polymers: from synthesis to practical applications. Polymers 8(4):118. https://doi.org/10.3390/polym8040118

3. Hatchett DW, Josowicz M (2008) Composites of intrinsically conducting polymers as sensing nanomaterials. Chem Rev 108(2):746-769. https://doi.org/10.1021/cr068112h

4. Aldissi M (1993) In intrinsically conducting polymers: an emerging technology, vol 246. Springer, Dordrecht. https://doi.org/10.1007/ 978-94-017-1952-0

5. Evtugyn G, Porfireva AP, Hianik T (2015) In: Tiwari A, Patra HK, Turner A (eds) Advanced bioelectronics materials. Wiley, Hoboken, pp 89-185

6. Zhou DD, Cui XT, Hines A, Greenberg RJ (2010) In: Zhou DD, Greenbaum E (eds) Implantable neural prostheses 2: techniques and engineering. Springer, New York, pp 217-252

7. Balint R, Cassidy NJ, Cartmell SH (2014) Conductive polymers: towards a smart biomaterial for tissue engineering. Acta Biomater 10(6):2341-2353. https://doi.org/10.1016/j.actbio.2014.02.015

8. Huang Z-B, Yin G-F, Liao X-M, Gu J-W (2014) Conducting polypyrrole in tissue engineering applications. Front Mater Sci 8(1):3945. https://doi.org/10.1007/s11706-014-0238-8

9. Zhomg L, Xiao S, Hu J, Zhu H, Gan F (2006) Application of polyaniline to galvanic anodic protection on stainless steel in H2SO4 solutions. Corros Sci 48(12):3960-3968. https://doi.org/ 10.1016/j.corsci.2006.04.019

10. Li J, Qian X, Wang L, An X (2010). Bioresources 5:712

11. Choi MR, Han TH, Lim KG, Woo SH, Huh DH, Lee TW (2011) Soluble self-doped conducting polymer compositions with tunable work function as hole injection/extraction layers in organic optoelectronics. Angew Chem Int Ed 50(28):6274-6277. https://doi. org/10.1002/anie.201005031

12. Li YQ, Li K, Liu B (2012) In: Wong W-Y, Abd-El-Aziz AS (eds) Molecular design and applications of photofunctional polymers and material. RSC Publishing, London, pp 399-423

13. Malinauskas A, Malinauskiene J, Ramanavicius A (2005). Beilstein J Nanotechnol 16:51

14. Ansari R, Keivani MB (2006) Polyaniline conducting electroactive polymers thermal and environmental stability studies. E-J Chem 3(4):202-217. https://doi.org/10.1155/2006/395391

15. Mawad D, Mansfield C, Lauto A, Perbellini F, Nelson GW, Tonkin T, Bello SO, Carrad DJ, Micolich AP, Mahat MM, Furman J, Payne DJ, Lyon AR, Gooding JJ, Harding SE, Terracciano CM, Stevens MM (2016) Sci Adv 2

16. Jaymand M (2013) Recent progress in chemical modification of polyaniline. Prog Polym Sci 38(9):1287-1306. https://doi.org/10. 1016/j.progpolymsci.2013.05.015 
17. Wang G, Vivek R, Wang J-Y (2017) Polyaniline nanoparticles: synthesis, dispersion and biomedical applications. Mini-Rev Org Chem 14(1):56-64. https://doi.org/10.2174/ $1570193 X 14666161118114230$

18. Cho H, Kim M, Lee S, Hwang W (2016) Stable and conformable superhydrophilic surface fabricated via surface-initiated silicification on polyaniline nanofibers. Appl Surf Sci 367:432-437. https:// doi.org/10.1016/j.apsusc.2016.01.215

19. Cui Y, Wei QQ, Park HK, Lieber CM (2001) Nanowire nanosensors for highly sensitive and selective detection of biological and chemical species. Science 293(5533):1289-1292. https:// doi.org/10.1126/science.1062711

20. Molino P, Zhang B, Wallace GG, Hanks TW (2015). Biofouling 31:493

21. Chen Y, Kang ET, Neoh KG, Wang P, Tan KL (2000) Surface modification of polyaniline film by grafting of poly(ethylene glycol) for reduction in protein adsorption and platelet adhesion. Synth Met 110(1):47-55. https://doi.org/10.1016/S0379-6779(99)00257-X

22. Roberts MJ, Bentley MD, Harris JM (2002) Chemistry for peptide and protein PEGylation. Adv Drug Deliv Rev 54(4):459-476. https://doi.org/10.1016/S0169-409X(02)00022-4

23. Huang J, Kaner R (2004) A general chemical route to polyaniline nanofibers. J Am Chem Soc 126(3):851-855. https://doi.org/10. $1021 / \mathrm{ja} 0371754$

24. Xia H, Narayanan J, Cheng D, Xiao C, Liu X, Chan HS (2005) Formation of ordered arrays of oriented polyaniline nanoparticle nanorods. J Phys Chem B 109(26):12677-12684. https://doi.org/ 10.1021/jp0503260

25. Geng YH, Sun ZC, Li J, Jing XB, Wang XH, Wang FS (1999) Water soluble polyaniline and its blend films prepared by aqueous solution casting. Polymer 40(20):5723-5727. https://doi.org/10. 1016/S0032-3861(99)00134-2

26. Emoto K, Harris JM, VanAlstine JM (1996) Grafting poly(ethylene glycol) epoxide to amino-derivatized quartz: effect of temperature and ph on grafting density. Anal Chem 68(21):3751-3757. https:// doi.org/10.1021/ac960114m

27. Blomquist M, Bobacka J, Ivaska A, Levon K (2013) Electrochemical and spectroscopic study on thiolation of polyaniline. Electrochim Acta 90:604-614. https://doi.org/10. 1016/j.electacta.2012.11.134
28. Goswami S, Nandy S, Calmeiro TR, Igreja R, Martins R, Fortunato E (2016) Stress induced mechano-electrical writing-reading of polymer film powered by contact electrification mechanism. Sci Rep 6(1):19514. https://doi.org/10.1038/srep19514

29. Angelopolos M, Asturias GE, Ermer SP, Scherr EM, MacDiarmid AG, Akhtar M, Kiss Z, Epstein A (1988). Mol Cryst Liq Cryst 160: 151

30. Lahiff E, Woods T, Blau W, Wallace GG, Diamond D (2009) Synthesis and characterisation of controllably functionalised polyaniline nanofibres. Synth Met 159(7-8):741-748. https://doi. org/10.1016/j.synthmet.2008.12.029

31. Kalarickal NC, Rimmer S, Sarker P, Leroux J (2007) Thiolfunctionalized poly(ethylene glycol)-b-polyesters: synthesis and characterization. Macromolecules 40(6):1874-1880. https://doi. org $/ 10.1021 / \mathrm{ma} 062377 \mathrm{~g}$

32. Lahiff E, Scarmagnani S, Schazmann B, Cafolla A, Diamond D (2010) Covalent attachment of functional side-groups to polyaniline nanofibres. Int J Nanomanuf 5(1/2):88. https://doi.org/ 10.1504/IJNM.2010.029926

33. Bergman B, Hanks TW (2000) Spectroscopic, microscopic, and surface analysis of alkanethiol- and fluoroalkanethiol-modified conducting polymer thin films. Macromolecules 33(21):80358042. https://doi.org/10.1021/ma000659p

34. Feast WJ, Tsibouklis J, Pouwer KL, Groenendaal L, Meijer EW (1996). Polymer 37:5034-5035

35. Kang ET, Neoh KG, Tan KL (1998) Polyaniline: a polymer with many interesting intrinsic redox states. Prog Polym Sci 23(2):277324. https://doi.org/10.1016/S0079-6700(97)00030-0

36. Li X, Zhuang T, Wang G, Zhao Y (2008) Stabilizer-free conducting polyaniline nanofiber aqueous colloids and their stability. Mater Lett 62(8-9):1431-1434. https://doi.org/10.1016/j.matlet.2007.08.078

37. Han C-C, Hseih W-D, Yeh J-Y, Hong S-P (1999) Combination of electrochemistry with concurrent reduction and substitution chemistry to provide a facile and versatile tool for preparing highly functionalized polyanilines. Chem Mater 11(2):480-486. https://doi. org $/ 10.1021 / \mathrm{cm} 980684 \mathrm{y}$

38. Molino P, Zhang B, Wallace GG, Hanks TW (2013) Surface modification of polypyrrole/biopolymer composites for controlled protein and cellular adhesion. Biofouling 29(10):1155-1167. https:// doi.org/10.1080/08927014.2013.830110 